\title{
Diseño arquitectónico de parque biblioteca para contribuir al desarrollo sociocultural en el sector Cono Norte IV, distrito de Ciudad Nueva, 2018
}

\author{
Architectural design of a library park to contribute to sociocultural \\ development in the sector Cono Norte IV, district of Ciudad
} Nueva, 2018

\author{
${ }^{1}$ Josselin Clarita Tone Calizaya
}

ORCID: 0000-0002-9667-7870

\section{RESUMEN}

El presente trabajo de investigación tuvo como objetivo desarrollar el diseño arquitectónico de un parque biblioteca para contribuir al desarrollo sociocultural en el sector Cono Norte IV, distrito de Ciudad Nueva, 2018; con una solución espacial y funcional óptima, partiendo de un análisis de la problemática actual que presenta el distrito a nivel de infraestructura, con el fin de promover la descentralización del conocimiento, la inclusión social y contribuir con el nivel educativo del distrito. El presente trabajo de investigación es no experimental - descriptivo. En la parte metodológica se aplicaron tres instrumentos: encuesta, ficha de observación y ficha técnica. Asimismo, los resultados evidenciaron la carencia de infraestructura bibliotecaria y el mal estado de conservación de los parques del distrito. El desarrollo de esta investigación permitió comprobar la hipótesis y se concluyó que el diseño arquitectónico de un parque biblioteca es una alternativa de infraestructura bibliotecaria de encuentro ciudadano; donde niños, jóvenes y adultos pueden acceder a información física y virtual; además que se fomenta las actividades sociales, culturales, educativas y recreativas; contribuyendo con el desarrollo sociocultural del distrito.

Palabras clave: Desarrollo sociocultural, distrito, parque biblioteca.

\section{ABSTRACT}

The aim of this research was to develop the architectural design of the library park to contribute to sociocultural development in the Sector Cono Norte IV, District of Ciudad Nueva, 2018; with an optimal spatial and functional solution, based on an analysis of the current problems presented by the District at the infrastructure level, in order to promote the decentralization of knowledge, social inclusion and contribute to the educational level of the District. The present research is not experimental - descriptive. In the methodological part, three instruments were applied: survey, observation sheet and technical data sheet. Likewise, the results show the lack of library infrastructure and the poor state of conservation of the District's parks. The investigation allows to verify the hypothesis of the research; and it has concluded that the architectural design of the Library Park is an alternative library infrastructure for citizen encounters where children, youth and adults access physical and virtual information; social, cultural, educational and recreational activities are encouraged; in order to contribute to the socio-cultural development of the District.

Keywords: Sociocultural development, District, Library Park

Arquitecta. E-mail:josselintc.23@gmail.com 


\section{INTRODUCCIÓN}

"El Desarrollo de un país tiene relación directa con el nivel de educación de sus habitantes" (Saavedra, 2014, p.12). En el Perú, “el analfabetismo ha estado presente en las zonas rurales y urbanas marginales debido a la poca descentralización..." (Saavedra, 2014, p. 36). Asimismo, "de acuerdo al Informe del Programa de Naciones Unidas para el Desarrollo - Índice de Desarrollo Humano a nivel nacional, departamental, provincial y distrital 2007, el distrito de Ciudad Nueva es el menos favorecido en cuanto a alfabetismo, escolaridad, logro educativo e ingreso familiar per cápita" (Municipalidad Provincial de Tacna, 2015 - 2025, p. 18).

El objetivo del trabajo de investigación fue plantear un diseño arquitectónico de Parque Biblioteca como una alternativa de solución a la carencia del servicio bibliotecario, con una propuesta arquitectónica de características sociales, culturales, educativas y recreativas, que contribuya al desarrollo sociocultural en el sector Cono Norte IV del distrito de Ciudad Nueva.

La motivación de la presente investigación radica fundamentalmente en proponer una arquitectura que se integre con el área libre del parque, mejorar la calidad de vida, lograr la apropiación del espacio, propiciar la educación ciudadana, el acceso cultural y la identidad de la población.

La relevancia del presente trabajo de investigación está en la propuesta arquitectónica; que, en el ámbito recreativo, logre la conexión con la red de parques existentes; en lo educativo, logre la conexión con las unidades educativas de la zona; y en lo cultural, el sentido de pertenencia de la comunidad con su distrito.

El problema parte de la falta de intervención en calidad educativa y espacios públicos de interacción sociocultural; por lo tanto, se ve reflejado en el bajo nivel educativo, social y cultural de la población del sector Cono Norte IV del distrito de Ciudad Nueva.

El resultado esperado del estudio es que pueda ser utilizado en un futuro como un instrumento, para promover proyectos de desarrollo social, cultural y educativo, para el disfrute y apropiación del espacio por niños, jóvenes y adultos del distrito, además de su aplicación en otros distritos de la ciudad de Tacna. Asimismo, la investigación tiene como alcance espacial el escenario del sector Cono Norte IV del distrito de Ciudad Nueva, de la ciudad de Tacna; desarrollándose durante el año 2018.

\section{PLANTEAMIENTO DEL PROBLEMA}

\section{Formulación del problema}

¿De qué manera el diseño arquitectónico de un Parque Biblioteca contribuirá al desarrollo sociocultural, en el sector Cono Norte IV, distrito de Ciudad Nueva, 2018?

\section{Justificación}

La presente investigación se llevó a cabo en el sector Cono Norte IV del distrito de Ciudad Nueva porque vio la necesidad de dar solución al problema de desarrollo social, cultural y educativo sumado al déficit de espacios públicos idóneos, la falta de apropiación del espacio público, la inseguridad y sentido de pertenencia de la población con su distrito. Por lo tanto, el trabajo de investigación se justifica por las siguientes razones:

- En lo social: El beneficio será promover la igualdad social, convivencia, integración y participación ciudadana; para el desarrollo integral del distrito de Ciudad Nueva.

- En lo económico: Se buscará la sostenibilidad a través de las actividades de servicios para el mantenimiento mismo del parque biblioteca.

- En lo ambiental: El beneficio radica en su contribución con el medio ambiente a través de la vegetación y el control de residuos sólidos, en la búsqueda de la apropiación y recuperación del valor paisajístico del distrito.

- En lo educativo: Los objetivos son

- En lo cultural: Se busca impulsar actividades artísticas y preservar el patrimonio cultural del distrito.

- En lo temático: Más que brindar el préstamo 
de material de lectura, se impulsan las relaciones interpersonales, al proponer espacios donde el trabajo educativo y de aprendizaje sea menos individual y más colectivo.

En conclusión, la meta inmediata que se pretende alcanzar con la propuesta arquitectónica, es que se convierta en un referente sociocultural en el sector Cono Norte IV del distrito de Ciudad Nueva; con espacios sociales, culturales, educativos y recreativos.

\section{Objetivos}

\section{- Objetivo general}

Desarrollar el diseño arquitectónico de un parque biblioteca para contribuir al desarrollo sociocultural en el sector Cono Norte IV, distrito de Ciudad Nueva, 2018.

\section{- Objetivos específicos}

- Elaborar un análisis y diagnóstico de la situación en que se encuentra la infraestructura bibliotecaria y parques en el sector Cono Norte IV del distrito de Ciudad Nueva.

- Analizar y diagnosticar el desarrollo social y cultural en el sector Cono Norte IV del distrito de Ciudad Nueva.

- Identificar y seleccionar el terreno para elaborar el análisis y diagnóstico estableciendo las condicionantes y determinantes para el diseño de un parque biblioteca.

\section{Variables}

\section{- Variable independiente}

Diseño arquitectónico de un parque biblioteca

\section{- Variable dependiente}

Desarrollo sociocultural

\section{MARCOTEÓRICO}

\section{Definición de Parque Biblioteca}

"El Parque Biblioteca es un nuevo concepto de biblioteca pública llevados a ámbitos de desarrollo e integración social" (Palomino, 2016, p. 29). Se conciben como un espacio de enlace comunitario, proyecto social y generador de nuevas dinámicas empresariales, culturales, educativas y lúdicas al servicio de los ciudadanos (Rendón \& Herrera, 2008, p.45). El concepto de Parque Biblioteca fue desarrollado por la Alcaldía de Medellín a cargo del alcalde Sergio Fajardo Valderrama, y propuesto en el Plan de Desarrollo 2004 - 2007. Aparece como uno de los proyectos estratégicos de la línea 3, cuyo fin es "el fortalecimiento de las bibliotecas como centros integrales de desarrollo cultural y social" (Alcaldía de Medellín, 2004). Asimismo, el proyecto consiste en "convertir las bibliotecas de nivel barrial y zonal, en centros culturales y comunitarios, cuya localización se hará en centralidades que, complementadas con equipamientos de menor escala, servirán como dinamizadores de las actividades barriales, a partir de proyectos estratégicos territoriales" (p.109).

\section{Proyecto de Parque Biblioteca}

El proyecto de Parque Biblioteca es concebida como un espacio para el encuentro ciudadano.
"Por ello, su estrategia espacial debe permitir una relación interior - exterior que estimule el desarrollo de multiplicidad de eventos colectivos, también su espacialidad debe ser flexible, y una organización con una gran eficiencia funcional y proyectar espacios que permitan el desarrollo de eventos propios de la interacción social" (Peña, 2014,p.143)

\section{Objetivos de los parques bibliotecas}

Según Rendón \& Herrera (2008), en el 2005, la Alcaldía de Medellín estableció los siguientes objetivos de los parques bibliotecas:

- Contribuir al mejoramiento de la calidad de vida de las personas.

- Crear condiciones para el desarrollo urbano.

- Propiciar la convivencia ciudadana a través del fortalecimiento de las actividades comunitarias.

- Construir espacios culturales, recreativos y educativos para la formación de ciudadanía.

- Mejorar el acceso a la información y propiciar la educación de ciudadana. 


\section{Estrategia de los parques bibliotecas}

La estrategia sobre los parques bibliotecas "es situar espacios culturales, recreativos y educativos de calidad llamados Parque Biblioteca, en diferentes zonas periféricas de la ciudad y posteriormente dotarlos de contenidos, así como de actividades comunitarias que ayuden a fortalecer la convivencia y el sentimiento de identidad" (Fundación Kreanta, 2009, p.253) (Ver Tabla $1)$.

\section{Espacios principales del Parque Biblioteca}

Tabla 1. Espacios principales de los parques bibliotecas

\begin{tabular}{|c|c|}
\hline $\begin{array}{l}\text { Centros de } \\
\text { desarrollo } \\
\text { empresarial } \\
\text { zonal }\end{array}$ & $\begin{array}{l}\text { Tienen como objetivo apoyar el } \\
\text { emprendimiento, el desarrollo y la } \\
\text { consolidación microempresas en los } \\
\text { barrios de la ciudad. }\end{array}$ \\
\hline Salas de lectura & $\begin{array}{l}\text { Las salas disponen de libros de todas las } \\
\text { tendencias literarias, así como CD-ROM, } \\
\text { CD y vídeos. Se cuenta con salas de } \\
\text { lectura para niños y niñas y para adultos } \\
\text { adaptadas a la edad de los usuarios. }\end{array}$ \\
\hline $\begin{array}{l}\text { Sala de } \\
\text { navegación } \\
\text { virtual }\end{array}$ & $\begin{array}{l}\text { Estas salas cuentan con ordenadores con } \\
\text { conexión gratuita y permanente a Internet. }\end{array}$ \\
\hline Auditorio & $\begin{array}{l}\text { El auditorio está destinado a la } \\
\text { presentación de obras teatrales, musicales } \\
\text { y las diferentes manifestaciones artísticas } \\
\text { de la comunidad }\end{array}$ \\
\hline Sala mi barrio & $\begin{array}{l}\text { En esta se encuentra la memoria escrita, } \\
\text { fotográfica y filmica de la zona, para dar a } \\
\text { conocer las costumbres y tradiciones del } \\
\text { barrio. }\end{array}$ \\
\hline Ludoteca & $\begin{array}{l}\text { Se trata de un espacio para niños y niñas } \\
\text { de } 0 \text { a } 10 \text { años, donde los menores, en } \\
\text { compañía de una orientadora, establecen } \\
\text { relaciones con los demás y participan en } \\
\text { actividades que enriquecen su } \\
\text { imaginación, motricidad y sensaciones. }\end{array}$ \\
\hline $\begin{array}{l}\text { Sala de } \\
\text { exposiciones }\end{array}$ & $\begin{array}{l}\text { Esta sala está destinada a exhibir las } \\
\text { diferentes manifestaciones artísticas de la } \\
\text { zona, de la ciudad y del país. }\end{array}$ \\
\hline Servicios & $\begin{array}{l}\text { Cafetería, papelería, locales comerciales, } \\
\text { etc. }\end{array}$ \\
\hline
\end{tabular}

Fuente: Fundación Kreanta (21 de septiembre de 2009). Transfiriendo conocimiento, cultura, educación y ciudad. Obtenido de Cátedra Medellín Barcelona: http://www.catedramedellinbarcelona. org/catedra.php

\section{Definición de desarrollo sociocultural}

Según la Real Academia Española, lo sociocultural es lo "perteneciente o relativo al estado cultural de una sociedad o grupo social". Por consiguiente, "el término sociocultural se relaciona con los aspectos sociales, culturales, e intelectuales de una población, donde interactúan los individuos por sí mismos, con el entorno y otras comunidades". (Rivera, 2015, p.17)

Por su parte, Villar (2003) afirma "El desarrollo sociocultural es el cambio en las formas culturales gracias a la generación de nuevas tecnologías e instrumentos de mediación y su traspaso a las generaciones siguientes" (p. 386).

\section{MATERIAL Y MÉTODOS}

El diseño de investigación es no experimentaldescriptivo por el tiempo, por el conocimiento confiable que se quiere producir y porque se realizará una visualización del proyecto arquitectónico, mas no se podrá realizar la ejecución física. Asimismo, por el periodo de tiempo en el que se obtendrán todos los datos, y realizándose esto en un determinado momento, es una investigación trasversal.

\section{Población y muestra}

\section{Población}

Con respecto a la población, esta está comprendida por el sector Cono Norte IV del distrito de Ciudad Nueva, constituida por 12 499 habitantes; según la tasa de crecimiento poblacional de $2 \%$ anual del censo INEI $2007 \mathrm{y}$ Plan Urbano del Distrito de Ciudad Nueva 2010 -2015 (Ver tabla 2). 
Tabla 2. Proyecciones del crecimiento poblacional del distrito de Ciudad Nueva

\begin{tabular}{|cccc|} 
Año & $\begin{array}{c}\text { Tasa } \\
\text { anual } \\
(\%)\end{array}$ & $\begin{array}{c}\text { Población } \\
\text { Proyectada } \\
\text { del distrito }\end{array}$ & $\begin{array}{c}\text { Población } \\
\text { proyectada } \\
\text { del sector } \\
\text { Cono Norte } \\
\text { IV }\end{array}$ \\
\hline 2010 & $2 \%$ & 36325 & 10671 \\
2011 & $2 \%$ & 37051 & 10884 \\
2012 & $2 \%$ & 37792 & 11101 \\
2013 & $2 \%$ & 38547 & 11323 \\
2014 & $2 \%$ & 39317 & 11549 \\
2015 & $2 \%$ & 40103 & 11779 \\
2016 & $2 \%$ & 40905 & 12014 \\
2017 & $2 \%$ & 41723 & 12254 \\
2018 & $2 \%$ & 42558 & 12499 \\
2043 & $2 \%$ & 69822 & 20508 \\
\hline
\end{tabular}

Fuente: INEI, Censo, 2007.

Elaboración: Equipo Técnico Unidad Formuladora de la Municipalidad Distrital de Ciudad Nueva y autora.

\section{Muestra}

El tipo de muestra a emplear es el muestreo aleatorio, el tamaño de la muestra es de 96 encuestas.

\section{Técnicas e instrumentos}

Para la realización de la presente investigación se realizó la investigación documental y la investigación de campo.

\section{Investigación documental}

- Técnica: Consiste en la recolección de información relacionada al tema de investigación.

- Instrumentos: Para analizar la variable independiente Parque Biblioteca, se utilizó:

- Ficha de observación en tres parques del distrito de Ciudad Nueva.

- Ficha técnica del Pabellón de Biblioteca y Museo de Tacna.

- Agenda cultural de la Biblioteca Pública DDC Tacna mayo 2018.

- Asistencia de lectores a la Biblioteca Central de Tacna en el año 2017 y 2018.

- ESCALE - Estadística de la Calidad Educativa
Para analizar la variable dependiente: Desarrollo Sociocultural, se utilizó:

- INEI - Censos Nacionales 2007: XI de Población y VI de Vivienda.

- CODISEC - Comité Distrital de Seguridad Ciudadana del distrito de Ciudad Nueva, 2017.

\section{- Investigación de campo}

- Técnica: Consiste en la visita al sector de intervención, clasificándolo y registrándolo de acuerdo a un esquema.

- Instrumentos: Para analizar la variable independiente Parque Biblioteca, se utilizó:

- Ficha de observación de la biblioteca escolar emblemática I.E. Mariscal Cáceres

- Encuesta a la población sobre el servicio Bibliotecario en el sector Cono Norte IV del distrito de Ciudad Nueva

\section{RESULTADOS}

\section{Análisis y diagnóstico de la variable independiente}

Para el desarrollo del análisis y diagnóstico de la variable independiente referida al Parque Biblioteca, se consideró lo siguiente: La infraestructura de los parques y la infraestructura bibliotecaria en el sector Cono Norte IV del distrito de Ciudad Nueva.

\section{- Análisis de la variable independiente}

\section{Infraestructura de los parques}

En el sector Cono Norte IV del distrito de Ciudad Nueva se han identificado un total de 28 parques distritales, 3 son parques de escala zonal y 25 son parques de escala vecinal. Asimismo, se ha planteado fichas de observación para analizar las características de los parques en el distrito de Ciudad Nueva, seleccionando los siguientes parques, por las características representativas de las escalas: 2 parques distritales de escala zonal, el Parque del Niño (ver Tabla 3) y el Parque Turístico; y 1 parque vecinal ubicado entre la intersección de la calle 4 con la calle Desaguadero. Dichas fichas de observación toman en consideración los siguientes criterios: aspectos físicos, espaciales, funcionales y de sostenibilidad. 
Tabla 3. Ficha de observación del Parque del Niño

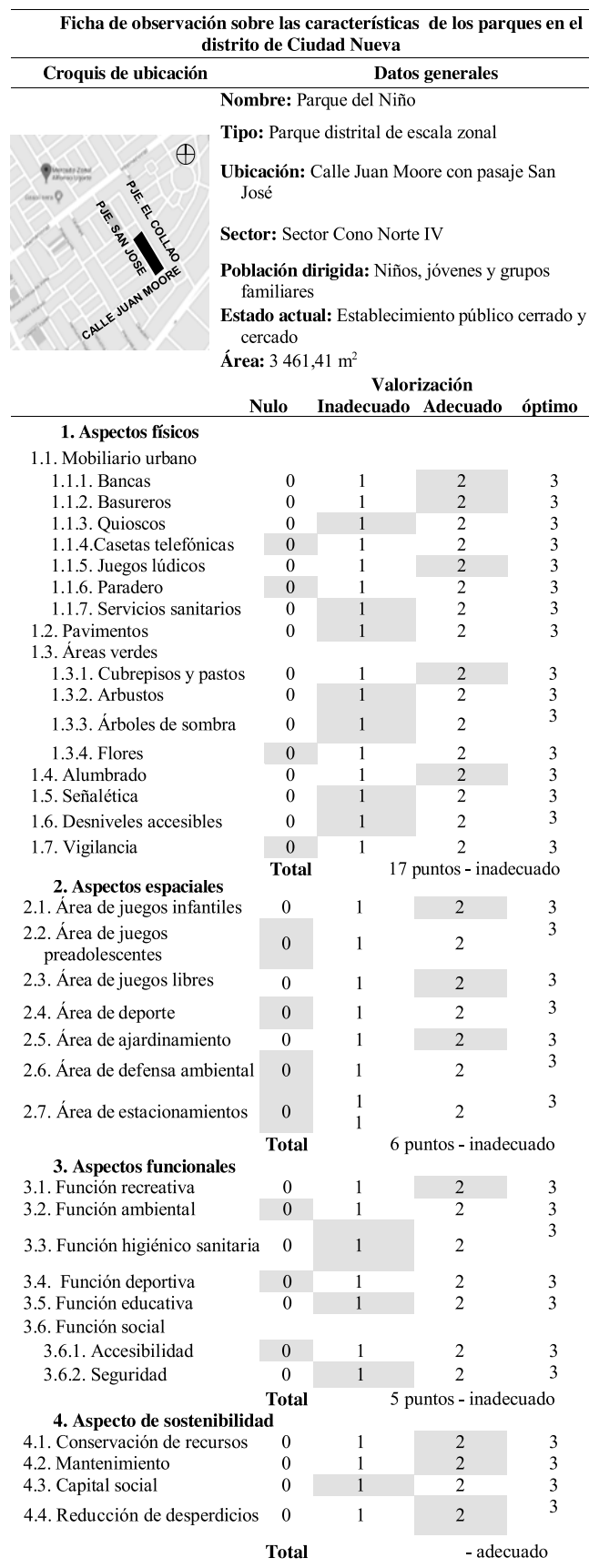

\section{Infraestructura bibliotecaria}

Para continuar con el desarrollo del análisis de la variable independiente, referida a la biblioteca, esto estará comprendido de la siguiente manera: análisis de las bibliotecas escolares del distrito (ver tabla 4); debido a la carencia de una infraestructura de biblioteca pública en el distrito se analizará la Biblioteca Pública - Dirección Desconcentrada de Cultura (DDC) de Tacna (ver tabla 5); y la encuesta sobre el servicio bibliotecario en el Sector Cono Norte IV del Distrito (ver figura 1).

Tabla 4. Ficha de observación de la biblioteca escolar emblemática I.E. Mariscal Cáceres

\begin{tabular}{lll|ll}
\hline \multicolumn{2}{c}{$\begin{array}{c}\text { Ficha de observación de la biblioteca escolar emblemática I.E. } \\
\text { Mariscal Cáceres }\end{array}$} & Valorización \\
\hline \multicolumn{1}{c}{ Requerimiento } & & & & \\
\hline 1. Los mobiliarios & 0 & 1 & 2 & 3 \\
$\begin{array}{l}\text { 1.1. Estanterías } \\
\text { 1.2. Mesas }\end{array}$ & 0 & 1 & 2 & 3 \\
1.3. Sillas & 0 & 1 & 2 & 3 \\
1.4. Muebles para exposición & 0 & 1 & 2 & 3 \\
1.4. Mostrador de atención & 0 & 1 & 2 & 3 \\
1.5. Trabajo interno & 0 & 1 & 2 & 3
\end{tabular}

\section{Los recursos}

2.1. Libros educativos

2.2. Obras literarias

2.3. Área de computadoras

2.4. Libros para inicial

2.5. Libros para primaria

2.6. Libros para secundaria

$\begin{array}{llll}0 & 1 & 2 & 3\end{array}$

$\begin{array}{llll}0 & 1 & 2 & 3\end{array}$

$\begin{array}{llll}0 & 1 & 2 & 3\end{array}$

$\begin{array}{llll}0 & 1 & 2 & 3\end{array}$

$\begin{array}{llll}0 & 1 & 2 & 3\end{array}$

$\begin{array}{llll}0 & 1 & 2 & 3\end{array}$

\section{La función}

3.1. Accesibilidad inmediata

$\begin{array}{llll}0 & 1 & 2 & 3\end{array}$

3.1. Ergonomía en los mobiliarios

$\begin{array}{llll}0 & 1 & 2 & 3\end{array}$

3.2. Espacios flexibles

3.3. Función iluminación natural

$\begin{array}{llll}0 & 1 & 2 & 3\end{array}$

3.4. Dimensión del espacio

$\begin{array}{llll}0 & 1 & 2 & 3\end{array}$

$\begin{array}{llll}0 & 1 & 2 & 3\end{array}$

\section{Función interna}

4.1. Área de trabajo interno

$\begin{array}{llll}0 & 1 & 2 & 3\end{array}$

4.2. Almacén de material documental

$\begin{array}{llll}0 & 1 & 2 & 3\end{array}$

13

Total

Inadecuado 
Tabla 5. Agenda cultural de la Biblioteca Pública DDC Tacna mayo 2018

\begin{tabular}{|c|c|}
\hline Fecha & Evento, lugar y hora \\
\hline $2018-05-07$ & $\begin{array}{l}\text { Ciclos de óperas en cine: concierto "Sinfonía de los } \\
\text { mil" de G. Mahler } \\
\text { Lugar: Auditorio "Virginia Lázaro Villarroel" } \\
\text { Hora: 7:30 h }\end{array}$ \\
\hline $2018-05-02$ & $\begin{array}{l}\text { Cuentacuentos: "Dino el cochino" } \\
\text { Lugar: Biblioteca Pública } \\
\text { Hora: } 10: 30 \mathrm{~h} \\
\text { Exposición de "Fotografía en escala" de Miguel } \\
\text { Coaquira } \\
\text { Lugar: Galería de Arte "Alberto Zeballos Franchi" } \\
\text { Hora: 7:00 h }\end{array}$ \\
\hline 2018-05-09 & $\begin{array}{l}\text { Ciclo de cine literario "El Principito" } \\
\text { Lugar: Biblioteca Pública } \\
\text { Hora: } 6: 30 \mathrm{~h}\end{array}$ \\
\hline $2018-05-14$ & $\begin{array}{l}\text { Ciclos de óperas en cine: "El lago de los cisnes" } \\
\text { Chailovski } \\
\text { Lugar: Auditorio "Virginia Lázaro Villarroel" } \\
\text { Hora: } 7: 30 \mathrm{~h}\end{array}$ \\
\hline $2018-05-16$ & $\begin{array}{l}\text { Café literario: Conociendo a mis literatos } \\
\text { Lugar: Biblioteca Pública } \\
\text { Hora: } 6: 30 \mathrm{~h}\end{array}$ \\
\hline $2018-05-21$ & $\begin{array}{l}\text { Ciclos de óperas en cine: "El castillo de barba } \\
\text { azul" de Bela Bartok } \\
\text { Lugar: Auditorio "Virginia Lázaro Villarroel" }\end{array}$ \\
\hline $2018-05-25$ & $\begin{array}{l}\text { Encuentro Cultural: "Muestra de catálogos de } \\
\text { exposiciones plásticas" } \\
\text { Lugar: Vestíbulo de la Biblioteca Pública } \\
\text { Hora: de } 8: 00 \mathrm{~h} \text { a } 6: 00 \mathrm{~h}\end{array}$ \\
\hline $2018-05-28$ & $\begin{array}{l}\text { Ciclos de óperas en cine: Sinfonía } \mathbf{N}^{\circ} \mathbf{2} \\
\text { "resurrección" de G. Mahler } \\
\text { Lugar: Auditorio "Virginia Lázaro Villarroel" } \\
\text { Hora: 7:30 h }\end{array}$ \\
\hline
\end{tabular}

La Figura 1 muestra que la mayoría de la población de niños, en un $56 \%$, mencionaron que no les agrada visitar las bibliotecas porque son pocas interactivas, seguido del $44 \%$ de la población de jóvenes, quienes indicaron que no le agradan por la falta de libros de su preferencia, mientras que el $50 \%$ de la población de adultos mencionó que son poco interactivos para sus hijos.

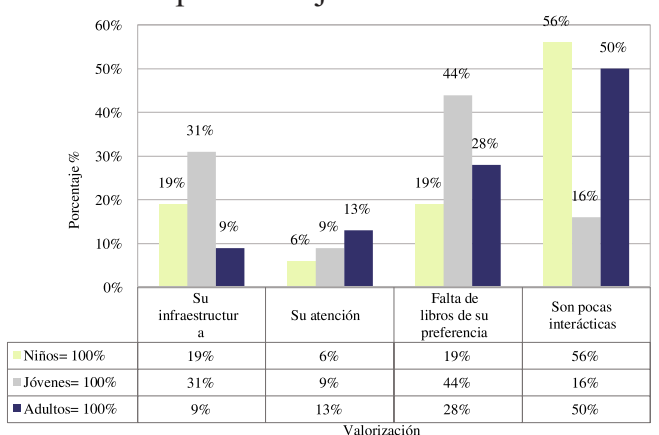

Figura 1. ¿Qué no le gusta de las bibliotecas?

Fuente: Encuesta realizada.

\section{- Diagnóstico de la variable independiente}

Diagnóstico situacional de los parques en el sector Cono Norte IV del distrito de Ciudad Nueva

La infraestructura de los parques en el sector Cono Norte IV del distrito de Ciudad Nueva presenta un deficiente servicio a la población, ya que en su mayoría no son accesibles por las barreras urbanísticas. Los mobiliarios urbanos como bancas y basureros, y luminarias no se encuentran en buen estado por la falta de mantenimiento; en otros casos, los espacios no funcionan como los quioscos, estacionamiento, servicios higiénicos. En consecuencia, no se puede considerar un atractivo espacio recreativo.

\section{Diagnóstico de la infraestructura bibliotecaria}

En la biblioteca escolar de la I.E. Mariscal Cáceres del distrito de Ciudad Nueva, existe poco interés por la lectura debido a las condiciones del ambiente en el cual se desarrolla, es decir, en la sala de laboratorio. Esto se da porque no cuenta con mobiliarios adecuados, espacio de atención, trabajo interno, y la dimensión correcta del espacio. Sin embargo, debido al recurso de los libros se percibe un pequeño interés por la lectura en los alumnos del nivel primario.

En la Biblioteca Pública de Tacna, existen iniciativas para difusión por la lectura, proponiendo a sus lectores una agenda cultural. Sin embargo, la desarticulación de la biblioteca con el Sistema Nacional de Bibliotecas del Perú conlleva a una desactualización del material bibliográfico; en consecuencia, existieron pocos lectores y disminución de usuarios lectores durante el año 2018 a comparación con el año anterior.

De la encuesta realizada a los pobladores en el sector Cono Norte IV del distrito de Ciudad Nueva, se percibe que existe una preocupación de la población adulta por la educación de sus hijos. Se obtuvo como resultado, que los adultos prefieren la zona para niños y talleres; mientras que los jóvenes prefieren zonas de internet, 
emprendimiento y apoyo a la población. Asimismo, los niños prefieren espacios para su desarrollo y zonas lúdicas.

\section{- Análisis de la variable dependiente}

Se enfocó en la población de acuerdo a los datos del INEI - Censos Nacionales 2007: XI de Población y VI de Vivienda; el Plan de Desarrollo Local Concertado del distrito de Ciudad Nueva 2017 - 2021 y el CODISEC Comité Distrital de Seguridad Ciudadana del distrito de Ciudad Nueva (Figura 2). Además de la encuesta realizada a la población sobre el desarrollo social y cultural del sector Cono Norte IV del Distrito (Figura 3), para conocer características educativas, culturales, y sociales de la población.

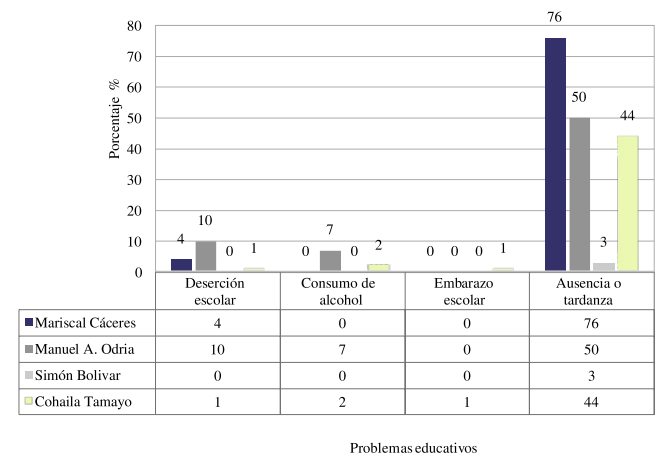

Emblemáticas del Distrito

Fuente: (CODISEC - Comité Distrital de Seguridad Ciudadana del Distrito de Ciudad Nueva, 2017)

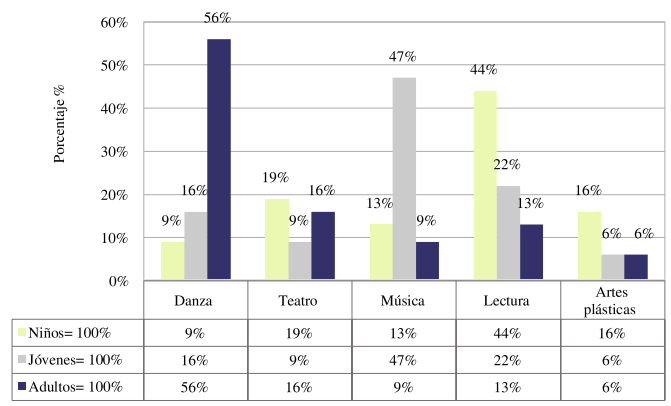

Figura 3. ¿Qué actividadešlociźútíturales practica o le gustaría practicar?

\section{- Diagnóstico de la variable dependiente}

Para mejorar el nivel educativo desde la niñez y generar la apropiación del espacio bibliotecario, es necesario proponer actividades complementarias y espacios que se ajusten a las necesidades de la población.

De la encuesta, la población mencionó que no ha participado de algún programa de desarrollo social, lo cual indica que las autoridades no logran llegar a las necesidades de los pobladores.

Respecto a los espacios recreativos, los niños prefieren los juegos lúdicos; los jóvenes, las losas deportivas; $y$ los adultos, prefieren el área de eventos. Por otro lado, la mayoría de la población encuestada mencionó que ha participado alguna vez de alguna actividad cultural. Asimismo, en relación a las actividades culturales que practican o les gustarían practicar, en el caso de los niños, ellos prefieren la lectura; los jóvenes prefieren la música; y los adultos prefieren la danza.

\section{CONCLUSIONES}

El diseño arquitectónico de un Parque Biblioteca contribuirá al desarrollo sociocultural en el sector Cono Norte IV del distrito de Ciudad Nueva, ofreciendo actividades sociales, culturales, educativas y recreativas.

El sector Cono Norte IV del distrito de Ciudad Nueva muestra carencia de espacios y servicios bibliotecarios que incentiven la lectura, además de un deficiente servicio de los parques para el esparcimiento, por ello, se requiere de un proyecto de Parque Biblioteca.

El sector Cono Norte IV del distrito de Ciudad Nueva presenta un bajo nivel de desarrollo sociocultural, por lo cual el diseño de un Parque Biblioteca es una alternativa como centro integral de encuentro ciudadano entre niños, jóvenes y adultos; que ayude a contrarrestar los bajos niveles de desarrollo social y cultural en los sectores menos favorecidos.

El terreno seleccionado más apropiado para desarrollar la adecuada propuesta arquitectónica de un Parque Biblioteca, se ubica entre la intersección de la avenida Mariano 
Necochea con la vía aprobada según PDU Tacna 2015 - 2025, adoptando los criterios de relación con el entorno, generando accesos y relación peatonal con los diferentes frentes.

\section{RECOMENDACIONES}

El diseño arquitectónico de un Parque Biblioteca debe constituirse como un modelo de infraestructura para contribuir al desarrollo sociocultural.

Debido a la carencia de infraestructura bibliotecaria y el deficiente servicio de los parques es necesario que la Municipalidad Distrital de Ciudad Nueva adopte iniciativas que generen la apropiación del espacio, a través de la integración de la arquitectura con el espacio abierto, el tipo de mobiliario flexible y el tratamiento cromático.

Debido a los bajos niveles de desarrollo sociocultural, las municipalidades deberían proponer en los sectores menos favorecidos una infraestructura de Parque Biblioteca, para proyectar una imagen arquitectónica que genere identidad de la comunidad, acceso a la información y a la educación.

Los proyectos destinados a parques bibliotecas deberán ser ubicados en la periferia de la ciudad, como centros integrales de desarrollo social y cultural, para niños, jóvenes y adultos.

\section{REFERENCIAS BIBLIOGRÁFICAS}

Alcaldía de Medellín. (2004). Plan de Desarrollo 2004-2007 "Medellín, compromiso de toda la ciudadanía". Medellín, Colombia: El Concejo de Medellín.

CODISEC - Comité Distrital de Seguridad Ciudadana del Distrito de Ciudad Nueva. (2017). Plan Local Seguridad Ciudadana Ciudad Nueva 2017. Tacna, Perú: Municipalidad Distrital de Ciudad Nueva y CODISEC.

Fundación Kreanta. (21 de septiembre de 2009). Transfiriendo conocimiento, cultura, educación y ciudad. Obtenido de Cátedra Medellín $\begin{array}{lllllllllll}\mathrm{B} & \mathrm{a} & \mathrm{r} & \mathrm{c} & \mathrm{e} & \mathrm{l} & \mathrm{o} & \mathrm{n} & \mathrm{a} & \text { : }\end{array}$ http://www.catedramedellinbarcelon a.org/catedra.php
INEI - Instituto Nacional de Estadística e Informática. (2007). Censo Nacionales 2007 XI de Población y VI Vivienda. Obtenido de Sistema de consulta de resultados Censales: http://censos.inei.gob.pe/cpv2007/tab ulados/default.asp

Municipalidad Distrital de Ciudad Nueva. (2010-2015). Plan Urbano del Distrito de Ciudad Nueva 2010-2015. Tacna, Perú: Equipo Técnico, Plan Urbano.

Municipalidad Provincial de Tacna. (2015 2025). Plan de Desarrollo Urbano de la Ciudad de Tacna 2015 - 2025. Tacna, Perú: MSOTTAC.

Palomino, H. (2016). Biblioteca Municipal de Comas (tesis de pregrado). Lima, Perú: Universidad Peruana de Ciencias Aplicadas, Facultad de Arquitectura.

Peña, M. (2014). Parques bibliotecas como estructuradores del espacio público de la ciudad de Colombia bajo los concepto de desarrollo sostenible. Módulo Arquitectura CUC (13), 129145.

Rendón, N. E., \& Herrera, R. (2008). Hacia una formación de usuarios de la formación en entornos locales. Información, Cultura y Sociedad $\mathrm{N}^{\circ} 19,35-62$.

Rivera, M. (2015). Producción de un Programa Comunitario con Enfoque Sociocultural en Radio Costa,Cantón Playas. Año 2015 (tesis de pregrado). La Libertad, Ecuador: Universidad Estatal Península de Santa Elena, Facultad de Ciencias Sociales y la Salud.

Saavedra, D. V. (2014). Biblioteca pública como centros de alfabetización funcional: Evaluación de programas en el Perú, utilizando el modelo yo sí puedo (tesis de pregrado). Lima, Perú: Universidad Nacional Mayor de San Marcos.

Villar, F. (23 de septiembre de 2003). Capítulo 7: Las Perspectivas Contextual y Sociocultural del Desarrollo. Obtenido de Psicología Evolutiva y Psicología de la Educación: http://www.ub.edu/dppsed/fvillar/pri ncipal/proyecto.html 